\title{
C9ORF72 hexanucleotide repeat expansions in the Italian sporadic ALS population
}

\author{
Mario Sabatelli ${ }^{\mathrm{a}, 1}$, Francesca Luisa Conforti ${ }^{\mathrm{b}, 1}$, Marcella Zollino ${ }^{\mathrm{c}, 1}$, Gabriele Mora ${ }^{\mathrm{d}, 1}$,
} Maria Rosaria Monsurrò ${ }^{\mathrm{e}}$, Paolo Volanti ${ }^{\mathrm{f}}$, Kalliopi Marinou ${ }^{\mathrm{d}}$, Fabrizio Salvi ${ }^{\mathrm{g}}$, Massimo Corboh , Fabio Giannini ${ }^{\text {, }}$, Stefania Battistini ${ }^{i}$, Silvana Penco ${ }^{\mathrm{j}}$, Christian Lunetta ${ }^{\mathrm{h}}$, Aldo Quattrone $^{\mathrm{b}}$, Antonio Gambardella ${ }^{\mathrm{b}}$, Giancarlo Logroscinok ${ }^{\mathrm{k}}$, Isabella Simone ${ }^{\mathrm{k}}$, Ilaria Bartolomei ${ }^{\mathrm{g}}$, Fabrizio Pisano ${ }^{1}$, Gioacchino Tedeschi ${ }^{\mathrm{e}}$, Amelia Conte ${ }^{\mathrm{a}}$, Rossella Spataro ${ }^{\mathrm{m}}$, Vincenzo La Bella ${ }^{\mathrm{m}}$, Claudia Caponnetto ${ }^{\mathrm{n}}$, Gianluigi Mancardi ${ }^{\mathrm{n}}$, Paola Mandich ${ }^{\mathrm{n}}$, Patrizia Sola ${ }^{\mathrm{o}}$, Jessica Mandrioli ${ }^{\mathrm{o}}$, Alan E. Renton ${ }^{\mathrm{p}}$, Elisa Majounie ${ }^{\mathrm{q}}$, Yevgeniya Abramzon ${ }^{\mathrm{p}}$, Francesco Marrosur ${ }^{\mathrm{r}}$, Maria Giovanna Marrosus, Maria Rita Murrus, Maria Alessandra Sotgiu', Maura Pugliatti ${ }^{u}$, Carmelo Rodolicov ${ }^{\mathrm{v}}$, the ITALSGEN Consortium², Cristina Moglia ${ }^{\mathrm{w}}$, Andrea Calvow $^{w}$, Irene Ossola ${ }^{\mathrm{x}}$, Maura Brunetti ${ }^{\mathrm{x}}$, Bryan J. Traynor ${ }^{\mathrm{p}, 1}$, Giuseppe Borghero ${ }^{\mathrm{r}, 1}$, Gabriella Restagno ${ }^{\mathrm{x}, 1}$, Adriano Chiò ${ }^{\mathrm{w}, 1, *}$

${ }^{a}$ Neurological Institute, Catholic University and I.CO.M.M. Association for ALS Research, Rome, Italy

${ }^{b}$ Institute of Neurological Sciences, National Research Council, Mangone, Cosenza, and University of Magna Grecia, Catanzaro, Italy

${ }^{c}$ Molecular Genetics Laboratory, Catholic University of Rome, Rome, Italy

${ }^{d}$ Salvatore Maugeri Foundation IRCSS, Scientific Institute of Milan, Milan, Italy

${ }^{e}$ Department of Neurological Sciences, Second University of Naples, Naples, Italy

${ }^{f}$ Salvatore Maugeri Foundation IRCSS, Scientific Institute of Mistretta, Mistretta, Italy

${ }^{g}$ Centre for Diagnosis and Cure of Rare Diseases, Department of Neurology, Bellaria Hospital, Bologna, Italy

${ }^{h}$ Neuromuscular OnmiCenter, Serena Foundation, Milan, Italy

${ }^{i}$ Department of Neuroscience, Neurology Section, University of Siena, Siena, Italy

${ }^{j}$ Department of Laboratory Medicine, Medical Genetics, Niguarda Ca' Granda Hospital, Milan, Italy

${ }^{k}$ Department of Neuroscience, University of Bari, Bari, Italy

${ }^{l}$ Salvatore Maugeri Foundation IRCSS, Scientific Institute of Veruno, Veruno, Italy

${ }^{m}$ ALS Clinical Research Center, Bio.Ne.C., University of Palermo, Palermo, Italy

${ }^{n}$ Department of Neuroscience, Ophthalmology and Genetics, University of Genoa, Genoa, Italy

${ }^{o}$ Department of Neuroscience, Sant'Agostino - Estense Hospital, and University of Modena, Modena, Italy

${ }^{p}$ Molecular Genetics Unit, Laboratory of Neurogenetics, NIA, Bethesda, MD, USA

${ }^{q}$ Neuromuscular Diseases Research Unit, Laboratory of Neurogenetics, NIA, Bethesda, MD, USA

${ }^{r}$ Azienda Universitaria-Ospedaliera di Cagliari, and University of Cagliari, Cagliari, Italy

${ }^{s}$ Centro Sclerosi Multipla, Ospedale Binaghi, Università di Cagliari, Cagliari, Italy

${ }^{t}$ Department of Biomedical Sciences, University of Sassari, Sassari, Italy

${ }^{u}$ Department of Neuroscience, University of Sassari, Sassari, Italy

${ }^{v}$ Department of Neurosciences, Psychiatric and Anaesthesiological Sciences, University of Messina, Messina, Italy

${ }^{w}$ Department of Neuroscience, University of Turin, Azienda Ospedale, Università San Giovanni Battista of Turin, Turin, Italy

${ }^{x}$ Molecular Genetics Unit, Department of Clinical Pathology, A.S.O. O.I.R.M.- Sant'Anna, Turin, Italy

Received 6 January 2012; received in revised form 10 February 2012; accepted 11 February 2012

\footnotetext{
* Corresponding author at: University of Turin and Azienda Ospedale Universita San Giovanni Battista of Turin, Department of Neuroscience, Torino, Italy. Tel.: +39 0116335439; fax: +390116963487.

E-mail address: achio@usa.net (A. Chiò).

${ }^{1}$ These authors contributed equally to this work.

${ }^{2}$ See Appendix 1 for the other members of ITALSGEN.
} 


\begin{abstract}
It has been recently reported that a large proportion of patients with familial amyotrophic lateral sclerosis (ALS) and frontotemporal dementia (FTD) are associated with a hexanucleotide (GGGGCC) repeat expansion in the first intron of C9ORF72. We have assessed 1757 Italian sporadic ALS cases, 133 from Sardinia, 101 from Sicily, and 1523 from mainland Italy. Sixty (3.7\%) of 1624 mainland Italians and Sicilians and $9(6.8 \%)$ of the 133 Sardinian sporadic ALS cases carried the pathogenic repeat expansion. None of the 619 regionally matched control samples (1238 chromosomes) carried the expansion. Twenty-five cases (36.2\%) had behavioral FTD in addition to ALS. FTD or unspecified dementia was also detected in 19 pedigrees $(27.5 \%)$ in first-degree relatives of ALS patients. Cases carrying the C9ORF72 hexanucleotide expansion survived 1 year less than cases who did not carry this mutation. In conclusion, we found that C9ORF72 hexanucleotide repeat expansions represents a sizeable proportion of apparent sporadic ALS in the Italian and Sardinian population, representing by far the most common mutation in Italy and the second most common in Sardinia.
\end{abstract}

(C) 2012 Elsevier Inc. All rights reserved.

Keywords: Amyotrophic lateral sclerosis; C9ORF72; Frontotemporal dementia; Survival

\section{Introduction}

Amyotrophic lateral sclerosis (ALS) is a neurodegenerative disorder of the adult life characterized by a progressive loss of lower motor neurons at spinal and bulbar level and of upper motor neurons. Its course is invariably fatal within 3 to 5 years from onset. While $5 \%$ to $10 \%$ of cases is familial in nature (familial ALS), the majority of patients present as apparently sporadic (sporadic ALS).

Recently, we found that a large hexanucleotide (GGGGCC) repeat expansion in the first intron of C9ORF72, a gene located on chromosome 9p21, is pathogenic in a large proportion of patients with familial ALS and frontotemporal dementia (FTD) of Finnish and European ancestry (Dejesus-Hernandez et al., 2011; Renton et al., 2011). In these families, the genetic defect is dominantly transmitted; however, mutations of this gene have also been detected in a number of patients with apparently sporadic ALS or FTD (Dejesus-Hernandez et al., 2011; Renton et al., 2011). To date, little clinical information is available about the characteristics of apparently sporadic ALS patients carrying C9ORF72 hexanucleotide repeat expansions.

The aim of the present report was to provide a detailed genotype-phenotype description of sporadic ALS cases carrying the expansion including age at onset, gender, site of onset, cognitive status, and survival.

\section{Methods}

A total of 1757 Italian sporadic ALS cases have been collected thorough the Italian ALS Genetic Consortium (ITALSGEN), which is a collaborative effort between 15 ALS centers throughout the Italian peninsula and the Mediterranean islands of Sicily and Sardinia. Of these, 133 were of Sardinian ancestry, 101 were from Sicily, and the remaining 1523 were from mainland Italy. Of note, we previously reported the $C 9 O R F 72$ repeat expansion status for 465 of these patients in our previous report (Majounie et al., in press). All cases included in the present study had a negative family history for ALS and were negative for mutations in major ALS genes (SOD1, TARDBP, and FUS). All cases had a diagnosis of definite, probable, probable laboratory-supported, or possible ALS according to El Escorial revised criteria (Brooks et al., 2000).

Controls consisted of 402 neurologically healthy subjects of mainland Italian ancestry, 61 of Sicilian ancestry, and 156 of Sardinian ancestry.

The clinical history of patients found to carry the C9ORF72 expansion was systematically reviewed to identify details of the disease phenotype including gender, age of onset, disease duration, disease variant, details of family history, and the presence of cognitive impairment. Formal cognitive evaluation was not systematically performed in this series. In 66 nonexpanded cases the age at onset, the site of onset, and the survival were not known.

\subsection{C9ORF72 analysis}

A repeat-primed polymerase chain reaction (PCR) assay was used to screen the presence of the GGGGCC hexanucleotide expansion in the first intron of C9ORF72 as described in the original report (Renton et al., 2011). This assay rapidly and robustly determines whether a sample carries the repeat expansion, but does not measure the actual number of repeats in the expansion. ALS-associated pathological expansions in C9ORF72 have been defined as greater than 30 repeats.

\subsection{Statistical analysis}

Continuous variables (i.e., age at symptom onset) were compared with $t$ test and discrete variable (i.e., gender, site of onset) with $\chi^{2}$. Survival was calculated with Kaplan and Meier curves and compared with log-rank test, using as end point death or tracheostomy. For censored cases, the last day of follow-up was November 1, 2011. Multivariable analysis was performed with the Cox proportional hazards model (stepwise backward). The following variables were included in the model: age (included as continuous vari- 
able), gender (male vs. female), site of onset (bulbar vs. spinal), ancestry (Italian vs. Sardinian), and C9ORF72 status (expanded vs. not expanded). A $p$ value $<0.05$ was considered significant. All calculations were made with SPSS version 18 (IBM Corporation, Chicago, IL, USA). The study was approved by the ethical committees of the participating centers. All patients signed a written informed consent.

\section{Results}

\subsection{Frequency of C9ORF72 pathological repeat expansion in sporadic $A L S$}

We tested 1523 mainland Italian patients, 101 Sicilian patients, and 133 Sardinian patients diagnosed with sporadic ALS for the presence of the GGGGCC hexanucleotide repeat expansion of the C9ORF72 gene using a repeatprimed polymerase chain reaction assay. Fifty-five $(3.6 \%)$ of the mainland Italians carried the pathogenic repeat expansion, whereas $5(4.9 \%)$ of the 101 patients with Sicilian ancestry, and 9 (6.8\%) of the 133 Sardinian sporadic ALS cases were carriers. None of the 402 mainland Italian, 61 Sicilian, and 156 Sardinian control samples (1238 chromosomes) carried the expansion. The median number of repeats in mainland Italian and Sicilian controls was 3 (range, 0-23; interquartile range, 0-5), and in Sardinian controls was 4 (range, 0-12; interquartile range, 0-5; see Supplementary Fig. 1).

Evaluation of the pedigrees of sporadic ALS carrying the pathological expansion did not reveal other cases of ALS. However, FTD or unspecified dementia was detected in first-degree relatives of ALS patients in 19 pedigrees (27.5\%). Moreover, we identified first degree relatives with parkinsonism in 3 pedigrees, and with schizophrenia in 2 pedigrees.

\subsection{Genotype-phenotype correlation}

The clinical characteristics of the sporadic ALS cases carrying C9ORF72 expansion are reported in Supplementary Table 1 . Twenty-five cases $(36.2 \%)$ had FTD in addition to their motor dysfunction. In all cases, FTD presented with behavioral symptoms, while none of the cases had features consistent with semantic dementia or progressive nonfluent aphasia. Three cases had additional clinical symptoms of parkinsonism, with positive dopamine transporter (DAT) scan, and 3 had psychotic symptoms (hallucinations and delusions).

Gender distribution and site of onset were similar in mutated and nonmutated cases (Table 1). Cases with C9ORF72 expansion had a slightly lower median age at onset than those without the expansion (59.0 [interquartile range, 50.0-65.6]; range, 32.9-83.0, vs. 62.8 [interquartile range, 54.0-69.7]; range, 20.5-89.6) ( $p=0.002)$ (Fig. 1).

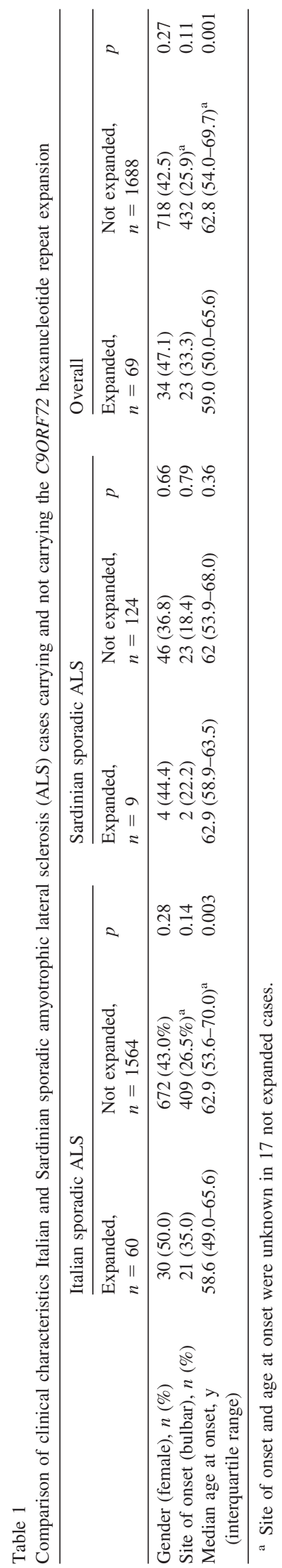




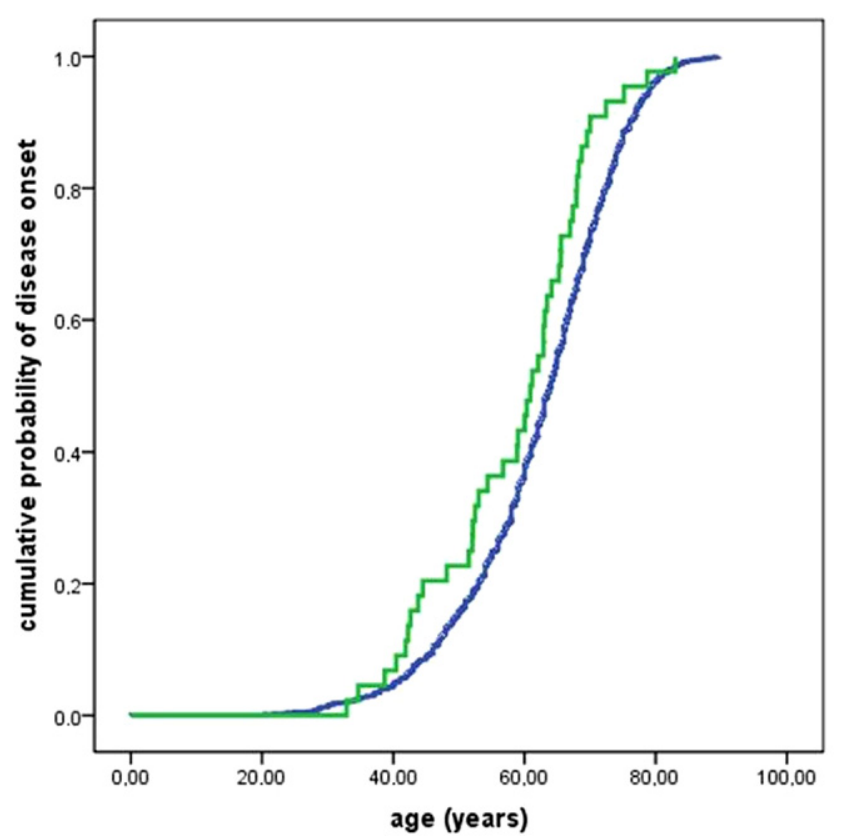

Fig. 1. Cumulative probability of disease onset according to the age of patients. Comparison between sporadic amyotrophic lateral sclerosis (ALS) patients with hexanucleotide repeat expansion of the C9ORF72 gene or unknown genetic mutation $(p=0.003)$.

\subsection{Tracheostomy-free survival}

Survival of cases carrying the C9ORF72 hexanucleotide expansion was 1 year shorter than that of cases who did not carry this mutation (C9ORF72 expanded cases, median survival time, 2.7 years; 95\% confidence interval [CI], 2.1-3.3; not expanded cases, median survival time, 3.6; 95\% CI, 3.3-3.8; $p=0.03$ ) (Fig. 2). The negative effect of C9ORF72 expansion on survival persisted in the Cox multivariate model (C9ORF72 status, not expanded vs. expanded hazard ratio, $1.79 ; 95 \% \mathrm{CI}, 1.26-2.98 ; p=0.008)$.

\section{Discussion}

C9ORF72 hexanucleotide repeat expansions have been detected in $3.7 \%$ of mainland Italian sporadic ALS of this series, which is representative of the Italian population, as it includes ALS cases originating from all Italian regions. Therefore, C9ORF72 hexanucleotide repeat expansions is by far the most common mutation in apparently sporadic ALS in the Italian population (Chiò et al., 2008; Corrado et al., 2010; Del Bo et al., 2009; Lai et al., 2011).

The frequency of Italian sporadic ALS carrying C9ORF72 hexanucleotide repeat expansion is lower than that reported in Finland (Renton et al., 2011), and in other north-European populations, such as Britons, Irish, and Germans, as well in Caucasian Americans (Dejesus-Hernandez et al., 2011; Gijselinck et al., 2012; Majounie et al., in press; Renton et al., 2011).

In sporadic ALS of Sardinian ancestry, C9ORF72 repeat expansions were more frequent than in mainland Italy, accounting for about $7 \%$ of cases, but they are less frequent than the A382T missense mutations of the TARDBP gene, which accounted for about $23 \%$ of sporadic ALS in the same population (Chiò et al., 2011; Orrù et al., 2012). The high frequency of the C9ORF72 mutation in Sardinia is likely to be related to the "magnification" of pathological genes in isolated populations, due to the so-called genetic drift phenomenon combined to the founder effect.

Sporadic ALS cases carrying the C9ORF72 hexanucleotide repeat expansion were phenotypically different from those not carrying the expansion. First, they were significantly younger, confirming previous observation in familial ALS patients carrying the same expansion (Chiò et al., 2012; Majounie et al., in press). Second, they had a more aggressive clinical course, with a median survival approximately 1 year less than cases without the expansion. According to the Cox multivariable analysis, the negative effect of C9ORF72 mutations on ALS survival is independent of age and site of onset. However, we did not include in the analysis the presence of FTD, a known negative prognostic factor in ALS (Elamin et al., 2011), which is known to have a higher frequency in ALS cases with C9ORF72 hexanucleotide repeat expansion than in those without this expansion (Dejesus-Hernandez et al., 2011; Renton et al., 2011). Finally, a third of cases with the expansion manifested cognitive impairment, consistent with previous reports of a high rate of FTD in this patient group.

The identification of the C9ORF72 expansion in appar-

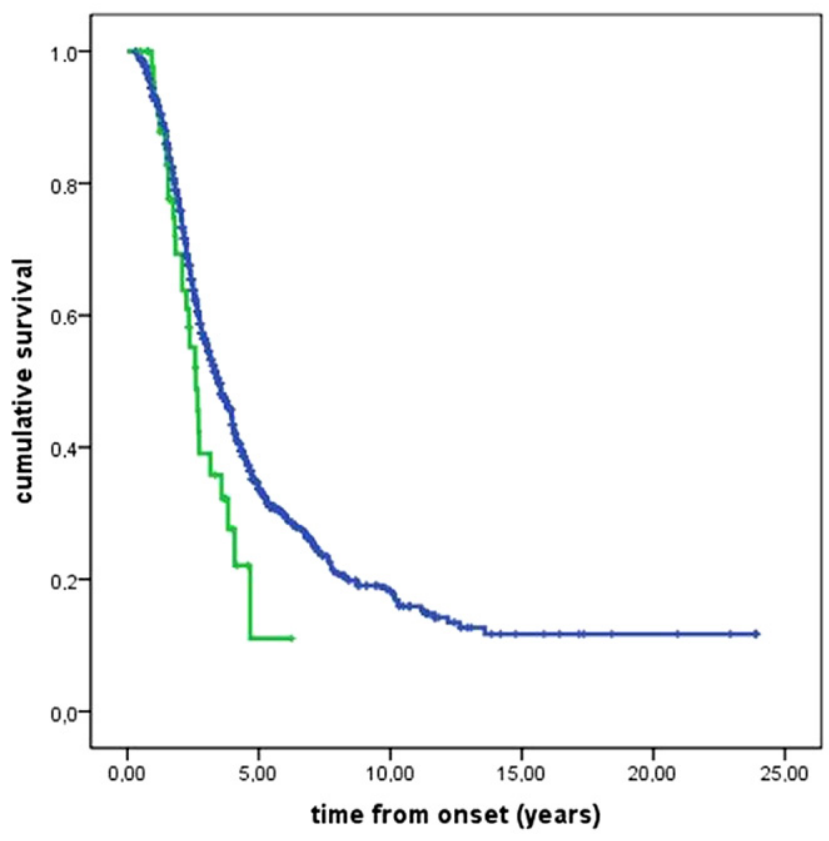

Fig. 2. Cumulative survival probability from time of disease onset. Comparison between sporadic amyotrophic lateral sclerosis (ALS) patients with hexanucleotide repeat expansion of the C9ORF72 gene and patients without known genetic mutation ( $p=0.023)$. Blue, unknown mutation; green, C9ORF72. Marks are censored patients. 
ently sporadic ALS may have different explanations, including poor diagnosis in the past, lack of knowledge of family history, mutation carriers in previous generations dying of other diseases prior to developing motor neuron degeneration, reduced penetrance of the gene, and even varying phenotypic manifestations among mutation carriers within the same family (Traynor and Singleton, 2009). An incomplete penetrance of C9ORF72 has been reported in a large multinational study (Majounie et al., in press). However, the likely familial nature of these apparently sporadic cases is supported by several findings. First, the gender distribution of the cases carrying the C9ORF72 repeat expansion in our sporadic series is not different from that expected for an autosomal dominant mutation. Second, the frequent identification of relatives with FTD or dementia in the patients' pedigrees support the notion that the C9ORF72 hexanucleotide expansion may manifest with different motor or cognitive phenotypes in the same pedigree. This observation implies that the definition of familial ALS should be enlarged to include patients with a positive family history for FTD, modifying the proposed criteria for definition of familial ALS (Byrne et al., 2011). Third, previous data indicate that ALS and FTD patients carrying the C9ORF72 mutation share a common haplotype and that all cases are in fact derived from a single founder (Mok et al., 2012).

In conclusion, we found that C9ORF72 hexanucleotide repeat expansions represent a sizeable proportion of apparent sporadic ALS in the Italian and Sardinian population, representing by far the most common mutation in Italy and the second most common in Sardinia, after the A382T missense mutation of the TARDBP gene. The high frequency of familial aggregates of ALS and dementia in the pedigrees of these apparently sporadic cases with the C9ORF72 mutation support the notion that most of these cases are in fact familial. An accurate study of the family history of ALS patients, as well the identification of comorbid FTD, may therefore be useful to clinically identify the possible carriers of this mutation.

\section{Disclosure statement}

Dr. Traynor reports that a patent is pending based on the discovery of the hexanucleotide repeat expansion of C9ORF72. The remaining authors disclose no conflicts of interest.

The study was approved by the ethical committees of the participating centers. All patients signed a written informed consent.

\section{Acknowledgements}

This work was supported in part by the Intramural Research Programs of the NIH, National Institute on Aging (Z01-AG000949-02), and NINDS. The work was also sup- ported by the Packard Center for ALS Research at Hopkins (BJT), the ALS Association (BJT, AC), Microsoft Research (BJT), Federazione Italiana Giuoco Calcio (BJT, AC), European Community's Health Seventh Framework Programme (FP7/2007-2013) under grant agreement 259867 (A.C), and ARISLA (BJT, AC).

\section{Appendix 1. Members of the ITALSGEN Consortium}

Stefania Cammarosano, M.D., Giuseppe Fuda, Antonio Canosa, M.D., Sara Gallo, M.D. (Department of Neuroscienze, University of Turin, Italy), Laura Papetti, Pharm.D. (Salvatore Maugeri Foundation, IRCCS, Scientific Institute of Milan, Italy), Giuseppe Lauria Pinter, M.D. (Department of Neurology, National Neurologic Institute Besta, Milan, Italy), Marco Luigetti, M.D. (Neurological Institute, Catholic University and I. Co MM Association for ALS Research, Rome, Italy), Serena Lattante, B.S., Giuseppe Marangi, M.D. (Molecular Genetics Laboratory, Catholic University of Rome, Italy), Tiziana Colletti, M.D. (ALS Clinical Research Center, Bio. Ne.C., University of Palermo, Italy), Claudia Ricci, M.D. (Department of Neuroscience, Neurology Section, University of Siena, Italy), Paola Origone, Ph.D. (Department of Neuroscience, Ophthalmology and Genetics, University of Genoa, Italy), Gianluca Floris, M.D., Antonino Cannas, M.D., Valeria Piras, M.D., Emanuela Costantino, M.D., Carla Pani, M.D. (Azienda Universitaria-Ospedaliera di Cagliari, and University of Cagliari, Italy), Leslie D. Parish, M.D., Paola Cossu, B.S. (Department of Neuroscience, University of Sassari, Italy), Giuliana Solinas, Ph.D., Lucia Ulgheri, Ph.D. (Department of Biomedical Sciences, University of Sassari, Italy), Anna Ticca, M.D. (AO San Francesco, Nuoro, Italy), Francesco Izzo, M.D., Anna Laiola, M.D., Francesca Trojsi, M.D. (Department of Neurological Sciences, Second University of Naples, Naples, Italy), Simona Portaro, M.D. (Department of Neurosciences, Psychiatric and Anaesthesiological Sciences, University of Messina, Italy and IRCCS Centro Neurolesi "Bonino-Pulejo", Messina, Italy), and William Sproviero, M.D. (Institute of Neurological Sciences, National Research Council, Mangone, Cosenza, Italy and University of Magna Gracia, Catanzaro, Italy).

\section{Appendix A. Supplementary data}

Supplementary data associated with this article can be found, in the online version, at doi:10.1016/j.neurobiolaging. 2012.02.011.

\section{References}

Brooks, B.R., Miller, R.G., Swash, M., Munsat, T.L., World Federation of Neurology Research Group on Motor Neuron Diseases, 2000. El Escorial revisited: revised criteria for the diagnosis of amyotrophic lateral sclerosis. Amyotroph. Lateral Scler. Other Mot. Neuron Disord. 1, 293-299. 
Byrne, S., Bede, P., Elamin, M., Kenna, K., Lynch, C., McLaughlin, R., Hardiman, O., 2011. Proposed criteria for familial amyotrophic lateral sclerosis. Amyotroph. Lateral Scler. 12, 157-155.

Chiò, A., Borghero, G., Pugliatti, M., Ticca, A., Calvo, A., Moglia, C., Mutani, R., Brunetti, M., Ossola, I., Marrosu, M.G., Murru, M.R., Floris, G., Cannas, A., Parish, L.D., Cossu, P., Abramzon, Y., Johnson, J.O., Nalls, M.A., Arepalli, S., Chong, S., Hernandez, D.G., ITALSGEN Consortium, Traynor, B.J., Restagno, G, 2011. A large proportion of ALS cases in Sardinia are due to a single founder mutation of the TARDBP geneLarge proportion of amyotrophic lateral sclerosis cases in Sardinia due to a single founder mutation of the TARDBP gene. Arch. Neurol. 68, 594-598.

Chiò, A., Borghero, G., Restagno, G., Mora, G., Drepper, C., Traynor, B.J., Sendtner, M., Brunetti, M., Ossola, I., Calvo, A., Pugliatti, M., Sotgiu, M.A., Murru, M.R., Marrosu, M.G., Marrosu, F., Marinou, K., Mandrioli, J., Sola, P., Caponnetto, C., Mancardi, G., Mandich, P., La Bella, V., Spataro, R., Conte, A., Monsurrò, M.R., Tedeschi, G., Pisano, F., Bartolomei, I., Salvi, F., Lauria Pinter, G., Simone, I., Logroscino, G., Gambardella, A., Quattrone, A., Lunetta, C., Volanti, P., Zollino, M., Penco, S., Battistini, S., the ITALSGEN Consortium, Renton, A.E., Majounie, E., Abramzon, Y., Conforti, F.L., Giannini, F., Corbo, M., Sabatelli, M. Clinical characteristics of familial ALS patients carrying the pathogenic GGGGCC hexanucleotide repeat expansion of the C9ORF72 gene. Brain 135, 784-793.

Chiò, A., Traynor, B.J., Lombardo, F., Fimognari, M., Calvo, A., Ghiglione, P., Mutani, R., Restagno, G., 2008. Prevalence of SOD1 mutations in the Italian ALS population. Neurology 70, 533-537.

Corrado, L., Del Bo, R., Castellotti, B., Ratti, A., Cereda, C., Penco, S., Sorarù, G., Carlomagno, Y., Ghezzi, S., Pensato, V., Colombrita, C., Gagliardi, S., Cozzi, L., Orsetti, V., Mancuso, M., Siciliano, G., Mazzini, L., Comi, G.P., Gellera, C., Ceroni, M., D’Alfonso, S., Silani, V., 2010. Mutations of FUS gene in sporadic amyotrophic lateral sclerosis. J. Med. Genet. 47, 190-194.

Dejesus-Hernandez, M., Mackenzie, I.R., Boeve, B.F., Boxer, A.L., Baker, M., Rutherford, N.J., Nicholson, A.M., Finch, N.A., Flynn, H., Adamson, J., Kouri, N., Wojtas, A., Sengdy, P., Hsiung, G.Y., Karydas, A., Seeley, W.W., Josephs, K.A., Coppola, G., Geschwind, D.H., Wszolek, Z.K., Feldman, H., Knopman, D.S., Petersen, R.C., Miller, B.L., Dickson, D.W., Boylan, K.B., Graff-Radford, N.R., Rademakers, R., 2011. Expanded GGGGCC Hexanucleotide Repeat in Noncoding Region of C9ORF72 Causes Chromosome 9p-Linked FTD and ALS. Neuron 72, 245-256.

Del Bo, R., Ghezzi, S., Corti, S., Pandolfo, M., Ranieri, M., Santoro, D., Ghione, I., Prelle, A., Orsetti, V., Mancuso, M., Sorarù, G., Briani, C., Angelini, C., Siciliano, G., Bresolin, N., Comi, G.P., 2009. TARDBP (TDP-43) sequence analysis in patients with familial and sporadic ALS: identification of two novel mutations. Eur. J. Neurol. 16, 727732.

Elamin, M., Phukan, J., Bede, P., Jordan, N., Byrne, S., Pender, N., Hardiman, O., 2011. Executive dysfunction is a negative prognostic indicator in patients with ALS without dementia. Neurology 76, 1263 1269.

Gijselinck, I., Van Langenhove, T., van der Zee, J., Sleegers, K., Philtjens, S., Kleinberger, G., Janssens, J., Bettens, K., Van Cauwenberghe, C., Pereson, S., Engelborghs, S., Sieben, A., De Jonghe, P., Vandenberghe, R., Santens, P., De Bleecker, J., Maes, G., Bäumer, V., Dillen, L., Joris, G., Cuijt, I., Corsmit, E., Elinck, E., Van Dongen, J., Vermeulen, S., Van den Broeck, M., Vaerenberg, C., Mattheijssens, M., Peeters, K.,
Robberecht, W., Cras, P., Martin, J.J., De Deyn, P.P., Cruts, M., Van Broeckhoven, C., 2012. A C9orf72 promoter repeat expansion in a Flanders-Belgian cohort with disorders of the frontotemporal lobar degeneration-amyotrophic lateral sclerosis spectrum: a gene identification study. Lancet Neurol. 11, 54-65.

Lai, S.L., Abramzon, Y., Schymick, J.C., Stephan, D.A., Dunckley, T., Dillman, A., Cookson, M., Calvo, A., Battistini, S., Giannini, F., Caponnetto, C., Mancardi, G.L., Spataro, R., Monsurro, M.R., Tedeschi, G., Marinou, K., Sabatelli, M., Conte, A., Mandrioli, J., Sola, P., Salvi, F., Bartolomei, I., Lombardo, F., ITALSGEN Consortium, Mora, G., Restagno, G., Chiò, A., Traynor, B.J., 2011. FUS mutations in sporadic amyotrophic lateral sclerosis. Neurobiol. Aging 32, e1-e4.

Majounie, E., Renton, A.E., Mok, K., Nicalou, N., Waite, A., Rollinson, S., Chiò, A., Restagno, G., Simon-Sanchez, J., van Swieten, J., Abramzon, Y., Johnson, J.O., Sendtner, M., Pamphlett, R., Orrell, R.W., Mead, S., Houlden, H., Rohrer, J.D., Morrison, K., Talbot, K., Ansorge, O., the Chromosome 9 ALS/FTD Consortium, the ITALSGEN Consortium, Englund, E., Borghero, G., McCluskey, L., Trojanowski, J.Q., van Deerlin, V.M., Schellenberg, G.D., Nalls, M.A., Drory, V., Brice, A., Drepper, C., Williams, N., Kirby, J., Shaw, P., Hardy, J., Singleton, A., Tienari, P.J., Heutink, P., Morris, H., Pickering-Brown, S., Traynor, B.J. C9ORF72 hexanucleotide repeat expansion in sporadic ALS and FTD around the world. Lancet Neurol. in press.

Mok, K., Traynor, B.J., Schymick, J., Tienari, P.J., Laaksovirta, H., Peuralinna, T., Myllykangas, L., Chiò, A., Shatunov, A., Boeve, B.F., Boxer, A.L., Dejesus-Hernandez, M., Mackenzie, I.R., Waite, A., Williams, N., Morris, H.R., Simón-Sánchez, J., van Swieten, J.C., Heutink, P., Restagno, G., Mora, G., Morrison, K.E., Shaw, P.J., Rollinson, P.S., Al-Chalabi, A., Rademakers, R., PickeringBrown, S., Orrell, R.W., Nalls, M.A., Hardy, J., 2012. The chromosome 9 ALS and FTD locus is probably derived from a single founder. Neurobiol. Aging 33, e3-e8.

Orrù, S., Manolakos, E., Orrù, N., Kokotas, H., Mascia, V., Carcassi, C., Petersen, M., 2012. High frequency of the TARDBP p.Ala382Thr mutation in Sardinian patients with amyotrophic lateral sclerosis. Clin. Genet. 81, 172-178.

Renton, A.E., Majounie, E., Waite, A., Simón-Sánchez, J., Rollinson, S., Gibbs, J.R., Schymick, J.C., Laaksovirta, H., van Swieten, J.C., Myllykangas, L., Kalimo, H., Paetau, A., Abramzon, Y., Remes, A.M., Kaganovich, A., Scholz, S.W., Duckworth, J., Ding, J., Harmer, D.W., Hernandez, D.G., Johnson, J.O., Mok, K., Ryten, M., Trabzuni, D., Guerreiro, R.J., Orrell, R.W., Neal, J., Murray, A., Pearson, J., Jansen, I.E., Sondervan, D., Seelaar, H., Blake, D., Young, K., Halliwell, N., Callister, J.B., Toulson, G., Richardson, A., Gerhard, A., Snowden, J., Mann, D., Neary, D., Nalls, M.A., Peuralinna, T., Jansson, L., Isoviita, V.M., Kaivorinne, A.L., Hölttä-Vuori, M., Ikonen, E., Sulkava, R., Benatar, M., Wuu, J., Chiò, A., Restagno, G., Borghero, G., Sabatelli, M., the ITALSGEN Consortium, Heckerman, D., Rogaeva, E., Zinman, L., Rothstein, J.D., Sendtner, M., Drepper, C., Eichler, E.E., Alkan, C., Abdullaev, Z., Pack, S.D., Dutra, A., Pak, E., Hardy, J., Singleton, A., Williams, N.M., Heutink, P., Pickering-Brown, S., Morris, H.R., Tienari, P.J., Traynor, B.J., 2011. A hexanucleotide repeat expansion in C9ORF72 is the cause of chromosome 9p21-linked ALSFTD. Neuron 72, 257-268.

Traynor, B.J., Singleton, A.B., 2009. What's the FUS! Lancet Neurol. 8, 418-419. 